\title{
Hemosiderotic Fibrolipomatous Tumor
}

National Cancer Institute

\section{Source}

National Cancer Institute. Hemosiderotic Fibrolipomatous Tumor. NCI Thesaurus. Code C121752.

A non-encapsulated, slow-growing, locally agg ressive subcutaneous tumor characterized by the presence of adipocytes, hemosiderin-laden spindle cells, hemosiderin-laden macrophages, osteoclast-like giant cells, and scattered chronic inflammatory cells. It usually arises from the dorsum of the foot, ankle, dorsum of the hand, thigh, calf, or cheek. It may recur if it is not completely excised but does not metastasize. 